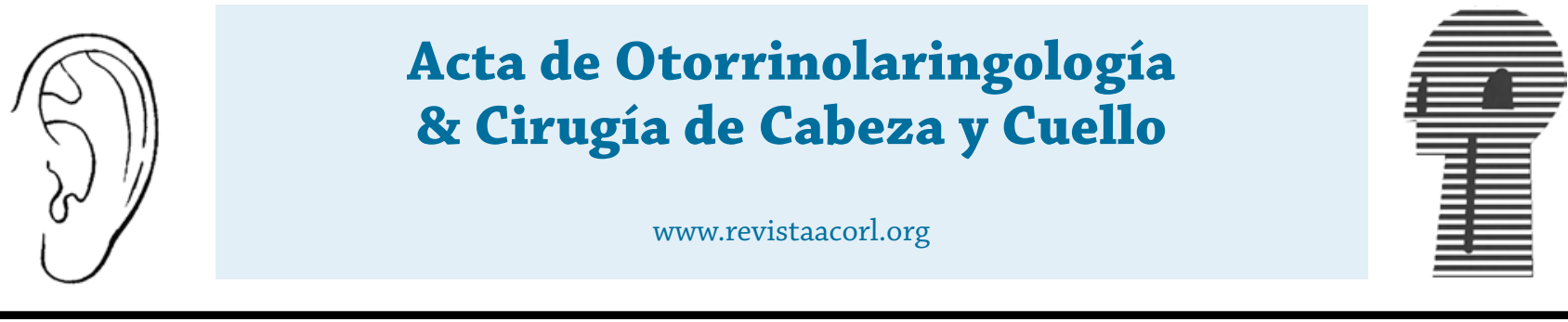

\title{
Evaluación de la percepción musical por estimulación acústica vs. eléctrica
}

\section{Evaluation of musical perception by acoustic vs. electrical stimulation}

Leonardo Elías Ordóñez-Ordóñez*, Felipe Osorio-Mejía**, Tatiana Garcia-Rey***, Claudia Paola González-Saboya**, Jorge Medina-Parra ${ }^{* * * *}$, Juan Alberto Ortiz-Obando ${ }^{* * * *}$, Estefany Catherine Hernández ${ }^{* * * * *}$, María Margarita SierraSandoval ${ }^{* * * * * *}$, Silvia Carolina Vanegas ${ }^{* * * * *}$, Néstor González-Marín ${ }^{* * * * * * *}$

\footnotetext{
* Otólogo-otorrinolaringólogo, profesor de la Fundación Universitaria Sanitas, Servicio de otorrinolaringología de la Clínica Universitaria Colombia. Bogotá, Colombia.

** Residente de IV año de otorrinolaringología, Fundación Universitaria Sanitas, Servicio de otorrinolaringología de la Clínica Universitaria Colombia. Bogotá, Colombia.

*** Otólogo-otorrinolaringólogo, Universidad Militar Nueva Granada. Bogotá, Colombia.

**** Epidemiólogo, Unidad de Investigaciones Fundación Universitaria Sanitas. Bogotá, Colombia.

***** Médico y cirujano, Clínica Universitaria Colombia. Bogotá, Colombia. Musicoterapeuta, Docente Universidad Nacional de Colombia

****** Servicio de fonoaudiología-audiología. Clínica Universitaria Colombia. Bogotá, Colombia.

******* Epidemiólogo, otorrinolaringólogo. Clínica Universitaria Colombia. Bogotá, Colombia.
}

Forma de citar: Ordóñez- Ordóñez LE, Osorio-Mejía F, Garcia-Rey T, González-Saboya CP, Medina-Parra J, Ortiz-Obando JA, Hernández EC, Sierra-Sandoval MM, Vanegas SC, GonzálezMarín N. Evaluación de la percepción musical por estimulación acústica frente a eléctrica. Acta otorrinolaringol. cir. cabeza cuello. 2020;48(1):62-68. Doi:10.37076/acorl.v48i1.514

INFORMACIÓN DEL ARTÍCULO

Historia del artículo:

Recibido: 10 de Diciembre de 2019

Evaluado: 25 de Abril de 2020

Aceptado: 21 de Mayo de 2020

Palabras clave (DeCS):

Implantes cocleares, música, percepción auditiva, pérdida auditiva sensorineural.

\section{RESUMEN}

Objetivo: evaluar las diferencias en la percepción musical entre la estimulación sonora eléctrica frente a la acústica. Materiales y métodos: se recolectó información de pacientes adultos con implante coclear unilateral, con oído contralateral sano o hipoacusia leve y con un tiempo de rehabilitación auditiva mayor a 12 meses. Se les aplicaron escalas de reconocimiento musical, de calidad de vida Glasgow Benefit Inventory (GBI), identificación de tono, ritmo, instrumentos y canciones. Resultados y conclusiones: se recolectaron datos de 6 pacientes con edades entre 37-73 años, promedio de tonos puros (PTA, por sus siglas en inglés) en oído sano $<40 \mathrm{~dB}$ y porcentaje de máxima discriminación (SDS, por sus siglas en inglés): $100 \%$ sin cambios en el postoperatorio. El oído implantado con un rango de PTA 
postoperatorio entre 40 y 58,3 dB y SDS entre 70-90\%. Se aplicó la escala GBI, en donde la mayoría de los pacientes obtuvieron resultados positivos con un rango entre +41 y -13 . La mayoría de los pacientes aumentaron la frecuencia de escucha musical post-implantación (4/6 pacientes). La mitad de los pacientes reportaron mejoría en el papel que desempeña la música en sus vidas. En el reconocimiento del tono y del ritmo en el oído implantado se encontraron resultados similares posterior a la implantación con respecto al oído sano, con medianas de 17/20 frente a $16 / 20$ en patrones tonales y $18,5 / 20$ frente a $18 / 20$ en patrones rítmicos. El reconocimiento de las canciones fue mejor con la letra que sin la letra en el oído implantado ( $8 / 8$ frente a $7 / 8$, respectivamente). El reconocimiento de instrumentos en el oído implantado fue 4,5/8 instrumentos frente a 7,5/8 en el oído sano.

\section{ABSTRACT}

Key words (MeSH):

Cochlear implants, music, auditory perception, sensorineural hearing loss.
Objective: To evaluate the differences in musical perception between electrical and acoustic sound stimulation. Materials and methods: Adult patients with unilateral cochlear implant, healthy or mild hearing loss in the contralateral ear and rehabilitation time greater than 12 months were evaluated. Musical recognition scales, quality of life Glasgow Benefit Inventory (GBI) and identification of tone, rhythm, instruments, and songs were applied. Results and conclusions: 6 patients were collected, ages between 37-73 years, healthy ear with a pure tone average (PTA) $<40$ $\mathrm{dB}$, speech discrimination score (SDS): $100 \%$ without changes in the postoperative period. The Implanted ear with average PTA between 40-58.3 dB and SDS between $70-90 \%$ post-implantation. The GBI scale was applied where most of the patients obtained positive results, with a range between +41 and -13 . Most of the patients improved the frequency of music-listening after implantation (4/6 patients). Half of the patients reported improvement in the role music plays in their lives. In the recognition of the tone and rhythm in the implanted ear, similar results were found after the implantation with respect to the healthy ear, with medians of $17 / 20 \mathrm{vs}$. $16 / 20$ in tonal patterns and $18.5 / 20$ vs. $18 / 20$ in rhythmic patterns. The recognition of the songs was better with the lyrics than without the lyrics in the implanted ear, with medians of $8 / 8$ vs. $7 / 8$. The recognition of instruments in the implanted ear was $4.5 / 8$ vs. $7.5 / 8$ in the healthy ear.

\section{Introducción}

La música es un fenómeno universal transcultural que hace parte de la vida de las personas como un método adicional de comunicación. La percepción y el disfrute por la música, siendo esta subjetiva y que para su valoración requiere de muchos factores que pueden ser relevantes como la educación musical previa al implante (1), tiene un gran impacto en la calidad de vida de las personas. Una de las opciones de rehabilitación en pacientes con hipoacusia neurosensorial severa a profunda es mediante la cirugía de implante coclear. El implante coclear es un excelente dispositivo para la rehabilitación del lenguaje, y en hipoacusias unilaterales con audición contralateral normal a leve sirve para reestablecer las ventajas de la binauralidad y/o para disminuir el tinnitus relacionado con la hipoacusia (2). A pesar de sus ventajas ampliamente estudiadas, una de las limitantes está en la percepción musical, ya que el paciente rehabilitado con implante coclear debido al cambio que existe en la estimulación acústica de un estímulo sonoro presente en una audición fisiológica normal es diferente comparada con la audición dada por una estimulación eléctrica como es el caso de los pacientes usuarios de implante coclear. Se ha demostrado que los implantes cocleares tienen muy buenos resultados para la percepción del lenguaje y adquisición del lenguaje hablado, pero está descrita una limitación del dispositivo para el procesamiento de melodías y el disfrute de la música. Se cree que la percepción musical y el disfrute de la música siguen siendo una limitante actual en estos dispositivos, sobre todo si se compara con su audición previa (3). Diferentes estudios han demostrado la capacidad de percibir con precisión los patrones rítmicos, pero con dificultades para reconocer patrones tonales y reconocer diferentes timbres $(4,5)$. En nuestro país faltan estudios sobre la música y el implante coclear, mientras que a nivel mundial, los estudios comparan el disfrute de la música post-implantación frente a su estado previo o frente a normooyentes (3-6), donde el paciente puede tener dificultades para recordar cómo era su disfrute musical. Por lo anterior y dadas las indicaciones en los últimos años de implantación en sorderas unilaterales, decidimos realizar un estudio comparando en el oído implantado vs el oído sano el disfrute de diferentes componentes de 
la música: ritmo, tono, timbre (identificación de instrumentos), reconocimientos de canciones populares en Colombia con y sin letra, y analizando cambios en la calidad de vida y experiencia musical post-implantación.

\section{Materiales y métodos}

Este es un estudio observacional comparativo con asignación no aleatoria de la muestra, realizado con pacientes de la Clínica Universitaria Colombia, en Bogotá D.C. Se recolectaron datos de 6 pacientes adultos con edades de 18 años o más, postlinguales, con implante coclear unilateral por hipoacusia neurosensorial severa a profunda, con oído contralateral sano o hipoacusia leve, tiempo de rehabilitación auditiva igual o mayor a 12 meses. Se excluyeron pacientes con algún grado de déficit cognitivo o que no aceptaran participar en el estudio. A cada uno de los pacientes se les aplicó escalas de hábitos musicales: Cuestionario Musical de Munich (7) y de calidad de vida (Glasgow Benefit Inventory, GBI) (8). Se realizaron pruebas de reconocimiento de tono y ritmo en cada uno de los oídos, reconocimiento de canciones populares en Colombia con y sin letra, y reconocimiento de instrumentos empezando por el oído implantado y continuando con el oído sano en una cabina acústica a una intensidad con la que el paciente se sintiera cómodo. Para su evaluación, se aisló el oído sano al retirar el implante coclear, y se aplicó oclusión contralateral y ruido blanco en el oído implantado.

\section{Hábitos musicales}

Se aplicó a cada uno de los participantes el Cuestionario Musical de Munich (7) para identificar los hábitos musicales previos y posteriores a la implantación coclear. De las preguntas contenidas en este cuestionario se evaluaron: la frecuencia de escucha musical, el papel que desempeña la música en sus vidas, el tiempo que dedican a escuchar música, el momento en el que empezaron a escuchar música posterior a la implantación, la capacidad para distinguir entre notas agudas y graves, la educación musical previa y si habían practicado la audición musical con el implante coclear.

\section{Evaluación de la calidad de vida mediante el cuestionario GBI}

Esta escala de 18 preguntas, con puntuaciones del 1 al 5 (1. Mucho peor, 2. Algo o un poco peor, 3. Sin cambio, 4. Algo o un poco mejor, 5. Mucho mejor), evalúa los cambios que se producen en el estado de salud de los pacientes después de una intervención otorrinolaringológica. En estas 18 preguntas se evalúan 3 subescalas: estado general de salud, cambios en el estado social y cambios en el estado físico. Los puntajes calculados van desde -100 (máxima sensación de empeoramiento) a +100 (máximo beneficio), siendo 0 ningún cambio (8).

\section{Identificación de tono y ritmo}

Para la identificación de tono y ritmo se utilizaron patrones rítmicos y tonales basados en el PMMA (Primary Measures of Music Audiation) una prueba estandarizada que evalúa pares de patrones cortos de tono y ritmo, en donde el paciente indica si el par de elementos suena igual o diferente y cuya ventaja es que no requiere de un entrenamiento musical formal para realizarla (6).

Se utilizaron 20 pares de patrones musicales de tono y ritmo con timbre de piano. Los patrones tonales contienen de dos a cinco notas variando de C4 $(260 \mathrm{~Hz})$ a F5 $(694 \mathrm{~Hz})$, y el ritmo a una misma frecuencia $(\mathrm{C} 5-520 \mathrm{~Hz})$.

\section{Identificación de instrumentos (timbre)}

Fueron evaluados 8 instrumentos escogidos con base en el estudio realizado por Lassaletta y colaboradores (2008), en donde el paciente debía identificarlos. Se utilizó un mismo patrón melódico de un tema musical reconocido en Colombia (Canción: La tierra del olvido; autor: Carlos Vives), reproducido en diferentes instrumentos: piano, guitarra, flauta, acordeón, trompeta y violín; y se reproducían adicionalmente 2 instrumentos de percusión: batería y guacharaca.

\section{Identificación de canciones}

De una lista de 20 canciones populares al inicio de las pruebas, el paciente escogía 8 que fueran reconocidas por él. Estas 8 canciones eran reproducidas inicialmente con letra y después sin letra, y el paciente debía mencionar el título o tararear la canción.

\section{Cuestionario de calidad del sonido}

Al finalizar las pruebas se evaluó en cada uno de los participantes la calidad del sonido de las canciones mediante una escala visual análoga (EVA) de 1-10. Dicha evaluación consistía en medir si se escuchaba natural-antinatural, agradable-desagradable, poco metálica-muy metálica y un poco resonante-muy resonante (7).

\section{Análisis estadístico}

Se describieron los resultados de las características de los pacientes en términos absolutos y relativos, se realizaron resultados gráficos para las características de sonido, para los resultados de la escala GBI y para el reconocimiento de instrumentos musicales. Se ejecutaron pruebas de rango con signo de Wilcoxon para analizar los resultados de reconocimiento y características musicales entre el oído implantado y el oído sano. Se estableció un valor de $\mathrm{p}<0,05$ para determinar la significancia estadística. Se ejecutaron las pruebas estadísticas en el programa STATA 15, y los gráficos se realizaron tanto en Microsoft Excel como en el programa estadístico mencionado. 


\section{Resultados}

Los sujetos estudiados fueron 2 pacientes masculinos y 4 pacientes femeninas con edades entre los 24 y 73 años, 2 implantados en el oído izquierdo y 4 implantados en el derecho. El tiempo de rehabilitación auditiva que recibieron los pacientes fue entre 12 y 45 meses desde el momento en que se activó el implante hasta el momento en que se realizaron las pruebas musicales. Diferentes dispositivos y procesadores fueron evaluados: 2 Advanced Bionics Naida, 2 Cochlear Nucleus 5, 1 Nurotron y 1 MED-EL Syncrhony. Las etiologías de la hipoacusia fueron variadas, en su mayoría hipoacusia súbita: 3 pacientes; otosclerosis: 1, otitis media crónica: 1 y trauma acústico: 1 . El porcentaje de máxima discriminación (SDS) postoperatorio en el oído implantado al momento de la realización de las pruebas musicales fue de $70-90 \%$, y el promedio tonal puro (PTA) entre 40 y 58,3 dB. En el oído sano, un PTA entre 5-35 dB y un SDS del $100 \%$ en todos los pacientes (Tabla 1).

\section{Hábitos musicales}

Todos los pacientes practicaron audición musical durante las terapias auditivas. La mayoría de los pacientes aumentaron la frecuencia de escucha musical postimplantación (4/6). La mitad de los pacientes reportaron una mejoría en el papel que desempeña la música en sus vidas. Los pacientes incrementaron la frecuencia de escucha musical (4/6) en comparación con el momento de la sordera previa al implante coclear. Los pacientes $(5 / 6)$ referían poder distinguir entre notas graves y agudas. Solamente un paciente había recibido educación musical, la cual se realizó en la infancia durante un período de al menos 3 años con entrenamiento en piano, flauta, cuatro y arpa.

\section{Evaluación de la calidad de vida mediante el cuestionario GBI}

Todos los pacientes respondieron el cuestionario, la mayoría de los pacientes reportaron mejoría (resultados positivos entre $+1 \mathrm{y}+100)$ con un rango entre +41 y -13 en el puntaje total, para un beneficio total de $+18,05$ (Tabla 2).

\section{Identificación de tono y ritmo}

En lo concerniente al reconocimiento del tono y ritmo, se encontraron resultados en el oído implantado que no distan de los valores encontrados en el oído sano, con medianas de $17 / 20$ frente a $16 / 20(\mathrm{p}=0,2878)$ para el tono, y de $18,5 / 20$ frente a $18 / 20(p=0,8273)$ para el ritmo. Inclusive en la mayoría de los pacientes $(4 / 6)$, el reconocimiento del tono fue mejor en el oído implantado.

Durante la evaluación de tonos, los pacientes que no habían tenido entrenamiento musical previo (5/6) obtuvieron respuestas erróneas (marcaron que los tonos F5-E5 a F5-D5 sonaban igual [2 semitonos de diferencia]) en el oído sano; curiosamente $3 / 5$ pacientes acertaron en el lado implantado. Lo mismo ocurrió con los patrones de notas C4-D4 a E4-D4 ( 2 semitonos de diferencia), en donde 3 pacientes acertaron con el uso del implante coclear. Todos los pacientes acertaron tanto en el oído sano como en el implantado el cambio de 1 semitono evaluado en el cambio de patrones de (C4-C4) a (C4-C\#4).

\section{Tabla 1. Información preoperatoria y postoperatoria de los pacientes implantados}

\begin{tabular}{|c|c|c|c|c|c|c|c|c|c|c|}
\hline 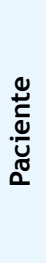 & 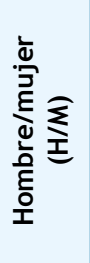 & 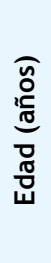 & $\begin{array}{l}\frac{0}{7} \\
\stackrel{0}{1} \\
\frac{\pi}{0} \\
\frac{0}{0} \\
\frac{0}{0} \\
\frac{0}{0}\end{array}$ & Tipo de implante & 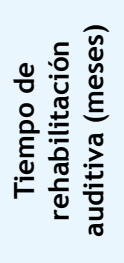 & $\begin{array}{c}\text { Etiología de la } \\
\text { hipoacusia }\end{array}$ & 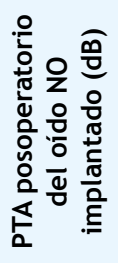 & 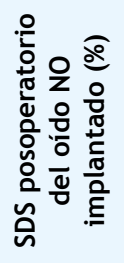 & 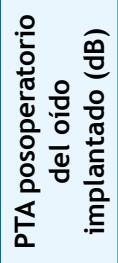 & 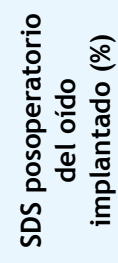 \\
\hline 1 & M & 51 & I & Advanced Bionics Naida & 24 & Sordera súbita & 5 & 100 & 40 & 70 \\
\hline 2 & M & 73 & D & Cochlear Nucleus 5 & 12 & Otosclerosis & 35 & 100 & 48,3 & 80 \\
\hline 3 & H & 37 & I & Advanced Bionics Naida & 30 & Sordera súbita & 5 & 100 & 58,3 & 80 \\
\hline 4 & M & 24 & D & Nurotron & 31 & OMC & 8,3 & 100 & 46,6 & 90 \\
\hline 5 & $\mathrm{H}$ & 64 & D & Cochlear Nucleus 5 & 45 & Trauma acústico & 15 & 100 & 41,6 & 80 \\
\hline 6 & M & 41 & D & Med-El (SYNCRHONY) & 27 & Sordera súbita & 13,3 & 100 & 40 & 70 \\
\hline
\end{tabular}

Tabla 2. Puntuación de la calidad de vida (Glasgow Benefit Inventory) posterior a la implantación coclear

\begin{tabular}{|l|c|c|c|c|c|c|c|}
\multicolumn{1}{|c|}{ Paciente } & $\mathbf{1}$ & $\mathbf{2}$ & $\mathbf{3}$ & $\mathbf{4}$ & $\mathbf{5}$ & $\mathbf{6}$ & $\mathbf{M e d i a n a}$ \\
\hline Puntaje total & 41,66 & 33,33 & 22,22 & 5,55 & 13,88 & $-13,88$ & 18,05 \\
\hline Subcategoría 1: cambios en el estado general & 50 & 33,33 & 54,16 & 4,16 & 12,5 & $-12,5$ & 22,915 \\
\hline Subcategoría 2: cambios en el soporte social & 66,66 & 50 & -50 & 16,66 & 0 & 16,66 & 16,66 \\
\hline Subcategoría 3: cambios en el estado físico & $-16,66$ & 16,66 & $-33,33$ & 0 & 33,33 & -50 & $-8,33$ \\
\hline
\end{tabular}


Tabla 3. Número de aciertos en el oído sano y en el oído implantado al evaluar diferentes características musicales

\begin{tabular}{|c|c|c|c|c|c|c|c|c|c|c|c|c|c|c|}
\hline \multirow[t]{2}{*}{ Paciente } & \multicolumn{2}{|c|}{1} & \multicolumn{2}{|c|}{2} & \multicolumn{2}{|c|}{3} & \multicolumn{2}{|c|}{4} & \multicolumn{2}{|c|}{5} & \multicolumn{2}{|c|}{6} & \multicolumn{2}{|c|}{ Mediana (\%) } \\
\hline & 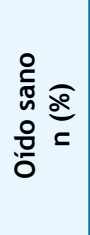 & 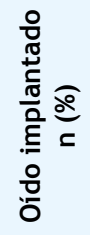 & 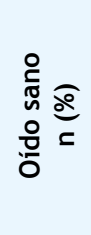 & 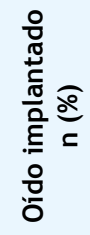 & 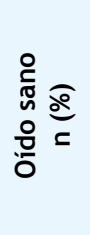 & 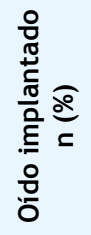 & 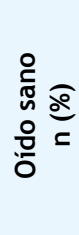 & 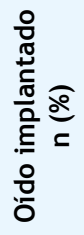 & 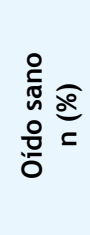 & 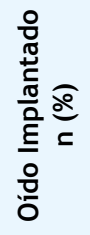 & 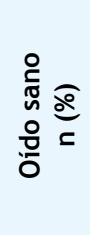 & 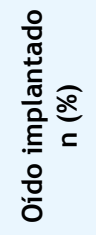 & 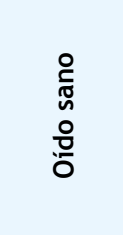 & 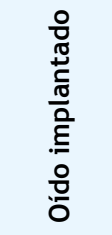 \\
\hline $\begin{array}{c}\text { Instrumentos } \\
(0-8)\end{array}$ & $8(100)$ & $7(87,5)$ & $6(75)$ & $3(37,5)$ & $8(100)$ & $3(37,5)$ & $8(100)$ & $6(75)$ & $7(87,5)$ & $4(50)$ & $7(87,5)$ & $5(62,5)$ & $7,5(93,75)$ & $4,5(56,25)$ \\
\hline Tono $(0-20)$ & $19(95)$ & $17(85)$ & $16(80)$ & $14(70)$ & $15(75)$ & $17(85)$ & $18(90)$ & 19 (95) & 15 (75) & $17(85)$ & $16(80)$ & 19 (95) & $16(80)$ & $17(85)$ \\
\hline Ritmo $(0-20)$ & $18(90)$ & $19(95)$ & $16(80)$ & 15 (75) & $18(90)$ & $18(90)$ & 19 (95) & $16(80)$ & $18(90)$ & 19 (95) & $20(100)$ & $20(100)$ & $18(90)$ & $18,5(92,5)$ \\
\hline $\begin{array}{c}\text { Canciones con } \\
\text { letra }(0-8)\end{array}$ & $8(100)$ & $5(62,5)$ & $8(100)$ & $8(100)$ & $8(100)$ & $8(100)$ & $8(100)$ & $8(100)$ & $8(100)$ & $8(100)$ & $8(100)$ & $8(100)$ & $8(100)$ & $8(100)$ \\
\hline $\begin{array}{c}\text { Canciones sin } \\
\text { letra }(0-8)\end{array}$ & $8(100)$ & $2(25)$ & $8(100)$ & $6(75)$ & $8(100)$ & $8(100)$ & $8(100)$ & $8(100)$ & $8(100)$ & $3(37,5)$ & $8(100)$ & $8(100)$ & $8(100)$ & $7(87,5)$ \\
\hline
\end{tabular}

Tabla 4. Calidad de la música a través del oído sano y del implante coclear

\begin{tabular}{|c|c|c|c|c|c|c|c|c|c|c|c|c|c|c|}
\hline Paciente & \multicolumn{2}{|c|}{1} & \multicolumn{2}{|c|}{2} & \multicolumn{2}{|c|}{3} & \multicolumn{2}{|c|}{4} & \multicolumn{2}{|c|}{5} & \multicolumn{2}{|c|}{6} & \multicolumn{2}{|c|}{ Mediana } \\
\hline Oído & 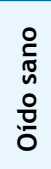 & 윰 $\frac{\stackrel{\circ}{\frac{0}{0}}}{\frac{\pi}{2}}$ & 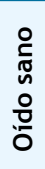 & 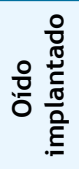 & $\begin{array}{l}0 \\
\stackrel{0}{c} \\
0 \\
0 \\
\circ \\
0 \\
0\end{array}$ & 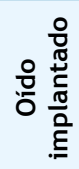 & 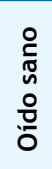 & 융 $\frac{\stackrel{0}{\frac{\pi}{\pi}}}{\frac{\pi}{\frac{\pi}{0}}}$ & 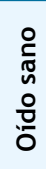 & 윰 $\frac{\stackrel{0}{\frac{0}{0}}}{\frac{\pi}{\frac{\pi}{0}}}$ & $\begin{array}{l}\circ \\
\stackrel{7}{0} \\
0 \\
\circ \\
\circ \\
0\end{array}$ & 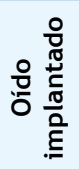 & $\begin{array}{l}\circ \\
\stackrel{0}{0} \\
\tilde{n} \\
\stackrel{0}{0} \\
0 \\
0\end{array}$ & 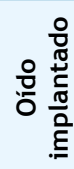 \\
\hline Natural & 10 & 4 & 8 & 2 & 8 & 6 & 10 & 10 & 8 & 7 & 10 & 1 & 9 & 5 \\
\hline Agradable & 10 & 4 & 8 & 7 & 8 & 7 & 10 & 10 & 9 & 8 & 10 & 5 & 9,5 & 7 \\
\hline Poco metálica & 10 & 2 & 9 & 7 & 8 & 7 & 10 & 10 & 8 & 6 & 10 & 3 & 9,5 & 6,5 \\
\hline Poco resonante & 10 & 4 & 7 & 5 & 9 & 8 & 10 & 10 & 7 & 6 & 10 & 3 & 9,5 & 5,5 \\
\hline
\end{tabular}

* Escala visual análoga (EVA): 1='antinatural', 'desagradable', 'muy metálica', 'muy resonante'; 10='natural', 'agradable', 'poco metálica', 'poco resonante'.

\section{Identificación de instrumentos (timbre)}

El reconocimiento de instrumentos en el oído implantado fue de 4,5/8 contra 7,5/8 del oído sano, siendo esta diferencia estadísticamente significativa $(\mathrm{p}=0,0269)$. El instrumento más difícil de identificar para el oído implantado fue el violín, ya que no fue reconocido por ninguno de los pacientes, seguido de la trompeta (1/6), el acordeón (3/6), la flauta y el piano $(4 / 6)$, la batería y la guacharaca (5/6), y por último la guitarra, que fue identificada por todos los pacientes (Figura 1).

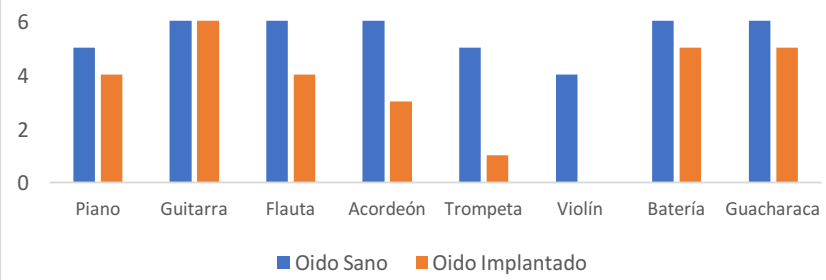

Figura 1. Reconocimiento de instrumentos musicales.

La única paciente que no identificó los instrumentos de percusión con el implante coclear tenía una caída importante en frecuencias graves de $65 \mathrm{~dB}$ a $250 \mathrm{~Hz}$ y $55 \mathrm{~dB}$ a 500 $\mathrm{Hz}$, normalizándose en frecuencias medias y agudas, lo que le permitió identificar la guitarra, la flauta y el acordeón. La mitad de los pacientes (3/6) tuvieron dificultades con los timbres agudos, y esto concuerda con las audiometrías al observar caídas con patrones descendentes en las frecuencias agudas.

\section{Identificación de canciones}

Todos los pacientes reconocieron las canciones populares con el oído no implantado. Al momento del reconocimiento de las canciones con el oído implantado, se encontró que fue mejor y más fácil reconocer las canciones con letra que sin letra, con medianas de $8 / 8$ frente a $7 / 8$, respectivamente $(\mathrm{p}=$ 0,0874). La mitad de los pacientes (3/6) pudieron reconocer las canciones con letra y sin letra por igual en el oído implantado. La otra mitad lo hizo mejor cuando las canciones tenían letra (Tabla 3).

\section{Cuestionario de calidad del sonido}

Al comparar el oído sano con el oído implantado, se encontró que la mayoría de los pacientes (5/6) escucharon las canciones de estas pruebas con una percepción más antinatural, menos agradable, más metálica y resonante en comparación con el oído no implantado. Uno de los pacientes no reportó cambios al evaluar estos ítems (Tabla 4; Figura 2). 


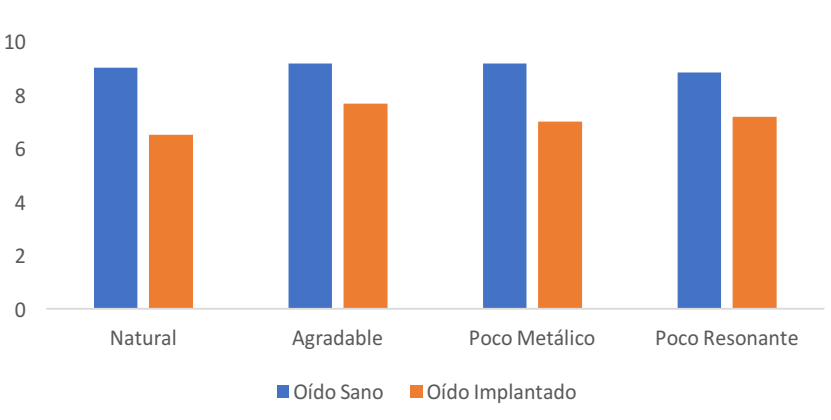

Figura 2. Calidad del sonido postimplante.

\section{Discusión}

Este estudio se diseñó para evaluar los resultados de algunas características musicales en un mismo paciente al escucharlas por estimulación acústica en comparación con la estimulación eléctrica. Los 6 pacientes estudiados, de diferentes edades, causas de hipoacusia, marcas de implante coclear, lateralidades, y la mayoría sin entrenamiento musical previo no tuvieron dificultades para realizar las pruebas, y los resultados fueron similares entre los pacientes evaluados a pesar de las diferencias mencionadas. Al igual que el habla, es clave practicar la audición de música con implantes cocleares en los pacientes para mejorar sus aptitudes musicales a pesar de no tener un entrenamiento musical previo.

Lassaletta y colaboradores (2008) encontraron que la discriminación de los ritmos es mejor que la de los tonos (3). En nuestro estudio no encontramos esta diferencia. El implante coclear, al igual que el oído sano, puede identificar patrones rítmicos y tonos simples. Se observó que el implante coclear puede incluso identificar cambios de tono tan pequeños como 1 semitono, cambios que para el oído no entrenado musicalmente es relativamente difícil, al contrario de lo reportado en estudios previos en donde la identificación de intervalos no era menos de 4 semitonos (5). Puede que los avances tecnológicos en el desarrollo de los implantes cocleares desde ese año hasta la actualidad hayan logrado esa mejoría en la discriminación de tonos.

En cuanto a la identificación de instrumentos, para la mayoría fue más fácil identificar la guitarra y los instrumentos de percusión (frecuencias graves y medias). Se observó que la identificación del timbre concuerda con la audiometría de los pacientes: al haber caídas neurosensoriales en frecuencias agudas, se dificulta la detección de timbres agudos; al haber caídas en frecuencias graves, se dificulta la identificación de los instrumentos de percusión.

La identificación de canciones con letra fue mejor que sin letra, probablemente, porque los implantes cocleares fueron diseñados para mejorar la comprensión del lenguaje hablado, y la tecnología en esta característica es avanzada. Este recurso hace que los pacientes puedan identificar las voces y las frases cantadas y reconocer qué canción está sonando más por su letra que por su melodía. Los mismos resultados se encuentran en otros estudios (3).
Como mencionan en su estudio Lassaletta y colaboradores (2008), la calidad del sonido parece ser fundamental en el disfrute de la música (3). En nuestros hallazgos, los pacientes encuentran disminuida la calidad del sonido al escucharlo con el implante coclear en comparación con el oído contralateral, factor que también encontramos fundamental para disfrutar la música.

Buyens y colaboradores (2014), al evaluar la preferencia de diferentes mezclas musicales, concluyeron que una pieza original de audio del "mundo real" no es agradable para los pacientes implantados; sin embargo, con algunas modificaciones en los volúmenes de algunos instrumentos podían encontrar la misma canción agradable (9). Esto concuerda con que al mejorar la calidad del sonido que llega al implante coclear, la música suena más placentera.

Buchman y colaboradores (2014) realizaron un ensayo aleatorizado prospectivo en el que evaluaron cómo la longitud de la inserción del electrodo afectaba el desempeño del implante coclear. Encontraron que la inserción más lejana del electrodo mejoraba el lenguaje; sin embargo, no existían diferencias entre los grupos de comparación en cuanto a la percepción de la música (10). En nuestro estudio no evaluamos la inserción del electrodo, pero al evaluar diferentes marcas de implantes cocleares que a su vez tienen diferentes longitudes y electrodos, concordamos en que no hay diferencias en cuanto a la percepción musical.

En general, la calidad de vida de los pacientes fue positiva posterior al implante coclear; sin embargo, probablemente por las diferencias en la percepción de la calidad del sonido entre los 2 oídos, escuchar la música de forma simultánea podría ocasionar distorsiones en la percepción binaural y en el disfrute general de la música. El resultado negativo (empeoramiento en la percepción de la calidad de vida) en la subescala cambios en el estado físico puede ser explicado por el hecho de que el paciente deje llevar un procesador de sonido extraño que genera una percepción de discapacidad, que se conoce como el estigma del sordo, y que también ha sido descrito con otros dispositivos de rehabilitación auditiva (11).

\section{Conclusiones}

Los resultados de este estudio muestran que, aunque el implante coclear puede ayudar a los pacientes a identificar diferentes patrones rítmicos, tonos, instrumentos y algunas canciones, la calidad del sonido no es lo suficientemente buena para que la escucha de la música sea tan agradable como en el oído normooyente. Dado que el implante coclear ha demostrado ser una ayuda importante para lograr una adecuada comunicación al identificar el lenguaje hablado, probablemente los implantes cocleares se valen de esta característica para identificar las canciones, dado que, al suprimir la letra de las canciones, se hace más difícil identificar las melodías. La frecuencia de escucha de música y el papel que esta representa en la calidad de vida mejora con el implante coclear en comparación con la hipoacusia previa. El disfrute de la 
música depende de muchos factores que pueden interferir con este. En el futuro deben realizarse estudios que evalúen diferentes pruebas musicales en pacientes con implante coclear unilateral en simultáneo (oído sano + oído implantado). La tecnología debe seguir avanzando para tratar de igualar la percepción musical por estimulación acústica.

\section{Conflicto de interés}

Los autores declaran no tener conflicto de interés.

\section{REFERENCIAS}

1. Harris RL, Gibson WP, Johnson M, Brew J, Bray M, Psarros C. Intra-individual assessment of speech and music perception in cochlear implant users with contralateral Cochlear ${ }^{\mathrm{TM}}$ and MED-ELTM systems. Acta Otolaryngol. 2011;131(12):12701278. doi:10.3109/00016489.2011.616225

2. Manrique M, Ramos A, Vernetta CA, Gil-Carcedo E, Lassaleta L, Sánchez-Cuadrado I, et al. Guía clínica sobre implantes cocleares. Acta Otorrinolaringol Esp. 2018. doi: 10.1016/j. otorri.2017.10.007

3. Lassaletta L, Castro A, Bastarrica M, Pérez-Mora R, Herrán B, Sanz L, et al. Percepción y disfrute de la música en pacientes poslocutivos con implante coclear. Acta Otorrinolaringol Esp. 2008;59(5):228-34. doi: 10.1016/S0001-6519(08)73300-4
4. Kong YY, Cruz R, Jones JA, Zeng FG. Music perception with temporal cues in acoustic and electric hearing. Ear Hear. 2004;25(2):173-185. doi:10.1097/01.aud.0000120365.97792.2f

5. Fujita S, Ito J. Ability of nucleus cochlear implantees to recognize music. Ann Otol Rhinol Laryngol. 1999;108(7 Pt 1):634- 640. doi:10.1177/000348949910800702

6. Gfeller K, Lansing CR. Melodic, rhythmic, and timbral perception of adult cochlear implant users. J Speech Hear Res. 1991;34(4):916- 920. doi:10.1044/jshr.3404.916

7. Brockmeier SJ, Fuster A. Cuestionario Música de Munich [Internet]. MED-EL. [acceso 19 de mayo de 2020]. Disponible en: https://s3.medel.com/downloadmanager/downloads/bridge us/Music_Listening/en-US/MUMU_Questionnaire_ES.pdf

8. Sánchez-Cuadrado IP. Validación de los cuestionarios de calidad de vida "Glasgow Benefit Inventory" y "Nijmegen Cochlear Implant Questionnaire" en pacientes con implante coclear [tesis]. Madrid: Universidad Autónoma de Madrid; 2015.

9. Buyens W, van Dijk B, Moonen M, Wouters J. Music mixing preferences of cochlear implant recipients: a pilot study. Int J Audiol. 2014;53(5):294- 301. doi:10.3109/14992027.2013.873955

10. Buchman CA, Dillon MT, King ER, Adunka MC, Adunka OF, Pillsbury HC. Influence of cochlear implant insertion depth on performance: a prospective randomized trial. Otol Neurotol. 2014;35(10):1773-1779. doi:10.1097/MAO.0000000000000541

11. David D, Werner P. Stigma regarding hearing loss and hearing aids: A scoping review. Stigma and Health. 2016;1(2):59-71. doi: $10.1037 /$ sah0000022 